Revue d'histoire de l'Amérique française

DAS REVUE D.HISTOIRE DE L'AMÉRIQUE FRANÇAISE

\title{
État, entrepreneurs, habitants et monopole : le " privilège " de la pêche au marsouin dans le Bas Saint-Laurent 1700-1730
}

\section{Alain Laberge}

Volume 37, numéro 4, mars 1984

URI : https://id.erudit.org/iderudit/304206ar

DOI : https://doi.org/10.7202/304206ar

Aller au sommaire du numéro

Éditeur(s)

Institut d'histoire de l'Amérique française

\section{ISSN}

0035-2357 (imprimé)

1492-1383 (numérique)

Découvrir la revue

Citer cet article

Laberge, A. (1984). État, entrepreneurs, habitants et monopole : le " privilège » de la pêche au marsouin dans le Bas Saint-Laurent 1700-1730. Revue d'histoire de l'Amérique française, 37(4), 543-556. https://doi.org/10.7202/304206ar d'utilisation que vous pouvez consulter en ligne. 


\title{
ÉTAT, ENTREPRENEURS, HABITANTS ET MONOPOLE: LE «PRIVILËGE» DE LA PÊCHE AU MARSOUIN DANS LE BAS SAINT-LAURENT 1700-1730*
}

\author{
ALAIN LABERGE \\ Étudiant au doctorat \\ Département d' histoire \\ Université York
}

\section{Introduction}

La pêche au marsouin au Canada voit le jour au début du XVIII ${ }^{\mathrm{e}}$ siècle alors que les malaises du secteur des fourrures et les difficultés d'implanter solidement la pêche de la morue dans le golfe Saint-Laurent poussent certains entrepreneurs à rechercher de nouveaux produits d'exportation. Les grands mammifères marins qui fréquentent l'estuaire du Saint-Laurent, notamment le marsouin, représentent une cible de choix car les produits qu'on en tire sont assez en demande.

Les peaux de marsouins sont sans doute vendues aux tanneurs de Québec en fonction du marché intérieur canadien ${ }^{1}$. L'huile de marsouin, estimée pour la consommation domestique et le corroyage des peaux, est davantage destinée au marché métropolitain. N'affirme-t-on pas que les producteurs «pourront toujours trouver le débit en France de 2000 barriques de ces huiles par chaque année» ${ }^{2}$. À un prix minimum de 55 à 60 livres la barrique et considérant qu'un marsouin donne enrivon une barrique d'huile, il est facile de concevoir l'optimisme animant les autorités coloniales et les entrepreneurs face à la pêche au marsouin ${ }^{3}$.

\footnotetext{
* Version remaniée d'une partie d'un mémoire de recherches intitulé: Une tentative de diversification économique en Nouvelle-France: la pêche dans le Bas Saint-Laurent, 1670-1750 (York University, 1983).

1 Transaction du 25 mai 1703, ANQQ, Greffe de Louis Chambalon. On y fait mention de peaux de marsouins chez les tanneurs Charest et Larchevesque.

2 «Projet de l'instruction (...)», 16 juin 1716, AC, C11A, 121: f. 71.

3 Les informations relatives aux prix de l'huile de marsouin proviennent, soit de la correspondance entre l'intendant et le ministre de la Marine, soit de mémoires ou d'états commerciaux, dans la série C11A. Elles sont peu fréquentes et imprécises et peuvent donc difficilement constituer une série statistique absolument fiable. Toutefois, on peut discerner une certaine évolution des prix: de 1700 à 1715, autour de 60 livres /barriques; entre 1715 et 1720 , soufflé par l'inflation, le prix peut atteindre 300 livres en cartes, dans les années 1720, stabilisation autour d'une centaine de livres /barrique, sans doute grâce à une forte demande en France; dans les années 1730 et 1740 , baisse à 80 puis à 65 livres /barrique, qu'on pourrait attribuer à une forte augmentation de la production d'huile de loup-marin qui affecte ainsi le prix de toutes les huiles de poisson du Canada. Tout cela reste cependant à vérifier et à compléter mais le manque chronique d'autres documents sur le commerce de l'huile ne contribue certes pas à éclairer cet aspect de la question.
} 
Entre 1700 et 1730, c'est la région de Rivière-Ouelle, sur la rive sud de l'estuaire, qui est le centre d'exploitation de la pêche au marsouin. Les marsouins la fréquentent du printemps à l'automne lors de leur migration annuelle dans l'estuaire. La température de l'eau et les espèces de petits poissons qui s'y trouvent conviennent tout à fait aux goûts du marsouin, tandis que le découpage du rivage, avec ses nombreuses pointes et anses, se prête fort bien à cette pêche littorale où la capture se fait dans un vaste parc de perches de bois plantées dans la vase en forme d'hémicycle ${ }^{4}$.

Pendant cette période, le développement de la pêche au marsouin dans la région de Rivière-Ouelle se caractérise par une série de tensions et de conflits entre différents groupes d'intérêts autour des droits d'exploitation. D'une part, le groupe des entrepreneurs, formé à l'origine de Charles Denys de Vitré, François Hazeur et Pierre Peire, cherche à obtenir des autorités coloniales le monopole de ce type de pêche dans l'estuaire du Saint-Laurent et tente de maintenir intact ce «privilège». D'autre part, des groupes d'habitants de la région s'opposent aux prétentions des entrepreneurs, soutenant que leur droit de pêche sur leur devanture éclipse tout autre droit d'exploitation, fût-il un monopole concédé par l'Etat.

Le problème était de taille et c'est à l'intendant que les parties s'en remettent pour trouver une solution. Très tôt, il devient évident que cette médiation va servir davantage à appuyer les visées économiques des intendants qu'à favoriser directement l'un ou l'autre des groupes en présence. Raudot et Bégon ont une préoccupation tenace: augmenter le produit de la pêche au marsouin pour en faire un secteur d'exportation fort et ainsi aider à la diversification de la chancelante économie canadienne du début du XVIII ${ }^{e}$ siècle. C'est la raison pour laquelle les décisions des intendants jouent constamment la carte du compromis et cherchent à éliminer les querelles qui nuisent à la pêche. Mais leurs ordonnances, par leur teneur même, minent graduellement le monopole des entrepreneurs, ouvrent la voie aux initiatives des habitants de la région et influencent considérablement le destin de la pêche au marsouin à Rivière-Ouelle après 1730 .

\section{I- Monopole et contestations, 1700-1720}

C'est en 1701 que les sieurs de Vitré, Hazeur et Peire obtiennent $\mathrm{du}$ gouverneur et de l'intendant le privilège «d'establir aux Îsles de Camourasca et autres endroits du fleuve Saint-Laurent qu'ils jugeront

\footnotetext{
4 V.D. Vladykov, Chasse, biologie et valeur économique du Marsouin blanc ou Béluga (Delphinapterus leucas) du fleuve et du golfe Saint-Laurent (Québec, Département des pêcheries, 1944), 194 p.; au sujet des techniques voir M. Moussette, La pêche sur le Saint-Laurent. Répertoire des méthodes et des engins de capture ([Montréal], Boréal Express, [1979]), 146-150; il existe aussi aux ANQQ une série de cartes et de plans sur la pêche au marsouin (B-913-SaintLaurent-1728).
} 
convenables la pesche des marsouins $(\ldots) »^{5}$. Le privilège de 1701 couvre une période de tâtonnements. Les connaissances techniques ne sont pas encore parfaitement au point et les entrepreneurs cherchent toujours le site de pêche idéal. L'État lui-même ne semble pas trop saisir les implications d'un tel privilège. C'est peut-être la raison pour laquelle le privilège n'est que de cinq ans, alors que les associés l'avaient demandé pour une durée de dix ans. Quoi qu'il en soit, il n'y a pas d'accrochages entre les habitants et les entrepreneurs car ces derniers semblent confiner leurs activités aux Îles de Kamouraska où il n'y a aucun établissement. On peut même croire qu'une partie de la main-d'oeuvre vient de l'extérieur de la région ${ }^{6}$. Le point saillant de la période 1701-1705 est surtout la mort de Vitré en 1703 grâce à laquelle Hazeur et Peire ramènent le contrôle de la société entre leurs seules mains?

L'année 1705 marque le début d'une longue période de conflits qui va durer jusque dans les années 1720 . Pourtant, la position des entrepreneurs paraît plus solide que jamais. En avril 1705, ils se voient confirmer la continuation du privilège de 1701 pour quinze autres années ${ }^{8}$. La technique des parcs de perches est à peu près au point et les entrepreneurs ont réalisé que la pointe nord-est de l'embouchure de la Rivière-Ouelle est un site on ne peut plus propice pour tendre des pêches à marsouins ${ }^{9}$. Qui plus est, ils peuvent utiliser les habitants de la région comme main-d'oeuvre pour dresser les perches, amener les marsouins sur la grève et procéder aux diverses opérations de dépeçage, de fonte des graisses et de mise en barriques. Ce dernier point est d'une importance considérable pour les entrepreneurs qui s'évitent ainsi l'embauche, le transport, le logement et la subsistance d'employés qui, de toutes manières, ne travailleraient pas de façon constante à la pêche, les captures de marsouins étant irrégulières et s'étendant sur plusieurs mois. Hazeur et Peire profitent donc de la présence d'une communauté agricole autour du site de pêche qu'ils ont choisi. Deux mois après la confirmation de leur privilège, ils s'associent à un groupe d'habitants de la région ${ }^{10}$. Jacques Gagnon, Jean Delavoye, Etienne Bouchard, Pierre Soucy, Pierre Boucher et François Gauvin vont travailler à la

\footnotetext{
5 Sentence arbitrale, 12 mai 1703, ANQQ, Greffe de Louis Chambalon.

6 Acte d'arrangement, 21 oct. 1701, ANQQ, Greffe de François Genaple.

Transaction du 25 mai 1703, ANQQ, Greffe de Louis Chambalon.

8 Ordonnance de Vaudreuil et Beauharnois, 21 avril 1705, AC, C11A, 22: f. 201.

9 La pointe du nord-est de la Rivière-Ouelle va demeurer jusqu'au $\mathrm{XX}^{\mathrm{e}}$ siècle un endroit renommé pour la pêche au marsouin, au même titre que l'île-aux-Coudres. Cette pointe fut concédée à Nicolas Huot au XVII ${ }^{\mathrm{e}}$ siècle qui la vendit à Pierre Dancosse en 1697 (ANQQ, Greffe de Louis Chambalon, 10 juin 1697). Après la mort de Dancosse, la même année, sa veuve, MarieMadeleine Bouchard, se remarie avec François Gauvin (29 mai 1702). C'est pourquoi, dans les documents, on parle indifféremment de la terre de «la veuve Dancosse», de «la femme Bouchard», de «la femme de Gauvin».

10 Ordonnance de Raudot, 15 juillet 1707, ANQQ, Ordonnances des Intendants [dorénavant $O I$ ], v. 1: f. 122.
} 
pêche sur place contre une part du produit de celle-ci, part qui s'élève d'abord aux deux tiers puis aux trois quarts des huiles recueillies ${ }^{11}$.

Les termes de cette entente généreuse nous laissent supposer que Hazeur et Peire auraient voulu étouffer au départ toute contestation possible de leur monopole. Savaient-ils que les habitants possédaient un droit de pêche légitime sur leur devanture? Nous l'ignorons. Mais cette façon de traiter les habitants en les associant carrément à l'entreprise est révélatrice des objectifs des entrepreneurs. D'abord, éliminer toute concurrence sur le site en s'associant directement aux intéressés éventuels (en l'occurrence ici, François Gauvin, le mari de la veuve Dancosse à qui appartient la pointe du nord-est, et ses voisins), pour ensuite disposer d'une main-d'oeuvre fiable et motivée à faire un succès de l'entreprise. Bref, s'assurer le contrôle d'un des meilleurs sites de pêche en centralisant entre leurs mains toutes les activités de pêche au marsouin pouvant naître à la pointe du nord-est de la Rivière-Ouelle. En 1705, on peut croire que les entrepreneurs sont maitres de la situation et que leur privilège s'exercera sans coup férir.

Malgré toutes ces précautions, les entrepreneurs ne peuvent parvenir à dissimuler la principale faiblesse de leur exploitation de pêche au marsouin à Rivière-Ouelle: ils ne sont aucunement propriétaires des lieux. Dans les nombreuses procédures légales qui vont se succéder par la suite, ils désespèrent de voir l'intendant affirmer une fois pour toutes que le privilège de pêche est au-dessus d'un simple droit de propriété des grèves.

La plupart des habitants de Rivière-Ouelle et des seigneuries voisines possèdent effectivement le droit de pêche sur la devanture de leur concession, droit qui leur confère la propriété de la grève. Seul le seigneur peut s'en réserver la jouissance s'il l'indique dans les contrats de concession, ce que le seigneur de Rivière-Ouelle n'a pas pris soin de faire avec ses premiers censitaires. Les habitants de Rivière-Ouelle, associés ou non aux entrepreneurs, cherchent alors à réaliser un objectif précis, absolument contraire aux intérêts des entrepreneurs privilégiés: faire reconnaître la primauté du droit de pêche sur tout autre droit subséquent. Ce qui revient à dire que quiconque a le droit de pêche sur sa

\footnotetext{
11 Idem, 8 août 1707, ANQQ, OI, v. 1: f. 126. Cette ordonnance, qui règle le premier conflit entre habitants et entrepreneurs, nous renseigne sur certains arrangements contractés entre eux. Les conditions semblent rester assez uniformes par la suite. Les entrepreneurs fournissent les outils et ustensiles nécessaires (pinces, crochets, cuves, seaux, etc.). Également les entrepreneurs décident de ne plus fournir les barriques pour l'huile en compensation de la diminution de leur part du tiers au quart. En plus d'augmenter leur part des deux tiers aux trois quarts, les habitants obtiennent aussi d'en disposer librement. Il est vraisemblable toutefois de penser que cette part doit être rachetée par les entrepreneurs eux-mêmes, bien qu'on n'ait aucune trace de ce type de marché. On ne sait trop non plus ce qu'il advient des peaux. Sans doute sont-elles incluses dans le partage mais cela est passé sous silence dans les documents d'avant 1720 .
} 
devanture peut décider de tendre ou non une pêche à marsouins et d'y associer qui il veut sans égard à aucun monopole ou privilège exclusif ${ }^{12}$.

Les entrepreneurs n'allaient pas tarder à voir à qui ils avaient affaire. Les premiers à se rebiffer contre le privilège et la tutelle qu'il leur impose sont ceux-là mêmes qui sont associés aux entrepreneurs, soit Jacques Gagnon «et consorts» comme les désigne l'intendant Raudot. En 1707, ces habitants forment une nouvelle société dont sont exclus les entrepreneurs. Malgré le refus de l'intendant de reconnaître leur société (suite aux plaintes de Hazeur et de Peire et après que Raudot se fût aperçu que Gagnon et consorts lui avaient caché la société existante avec les deux entrepreneurs) les habitants ne sortent pas les mains vides de ce geste audacieux. Outre des avantages matériels accrus (voir la note \# 11), le droit de pêche leur est reconnu sur leur devanture après que l'intendant en eût vérifié l'existence auprès du seigneur de RivièreOuelle $^{13}$. C'est là la première brèche au privilège de Hazeur et de Peire.

Par la suite, il semble s'être produit un relâchement de l'emprise du privilège. Hazeur meurt à l'été de 1708 et Peire, sans doute un peu déçu des résultats de la pêche au marsouin, s'occupe alors davantage de commerce avec les Antilles où il décède quelque temps après ${ }^{14}$. Dès la fin de 1708, profitant des circonstances et de son droit de pêche, François Gauvin baille la devanture de la pointe du nord-est de la RivièreOuelle à Louis Rouer d'Artigny pour neuf ans ${ }^{15}$. Il va s'écouler plus d'un an avant que les nouveaux représentants du privilège ne réagissent à cette intrusion. Mais au lieu de s'opposer à la présence de Rouer d'Artigny, Philippe Peire (pour son frère) et Joseph Riverin (pour les créanciers de la succession de Hazeur) décident de s'associer avec lui pour le restant de la durée du privilège, restaurant par le fait même une certaine cohésion à la direction de celui-ci ${ }^{16}$.

12 Cette situation est diamétralement l'inverse de celle qui prévaut à l'Île-aux-Coudres. Là, le Séminaire de Québec, seigneur de l'île, se réserve la jouissance des grèves et peut donc choisir lui-même, d'une part, les sites de pêche au marsouin et, d'autre part, les habitants qui y travailleront; d'où des opérations centralisées qui équivalent virtuellement à un monopole. Il est à noter cependant que le Séminaire commence à distribuer les terres quand les techniques de pêche au marsouin sont connues, contrairement à ce qui se passe à Rivière-Ouelle et aux alentours, lors des concessions des terres en bordure du fleuve entre 1670 et 1700 . Sur la pêche aux marsouins à l'Île-aux-Coudres, voir M. Lalancette, La seigneurie de l'Île-aux-Coudres au XVIII'me siècle, Thèse de M.A. non publiée (Université de Montréal, 1980), 224 p.

13 Ordonnance de Raudot, 13 juillet 1707, dans Édits, ordonnances royaux, déclarations et arrêts du Conseil d'État (...), 1854, [dorénavant EO], III: 419.

14 On ne connaît pas la date exacte du décès de Pierre Peire. Selon Bégon, il serait mort aux «Îles de l'Amérique» en 1708 (AC, C11A, 33: f. 134v., 12 novembre 1712). Par contre, selon Philippe Peire, le frère de l'entrepreneur, celui-ci est toujours vivant en 1710, bien qu'absent du Canada (ANQQ, Greffe de Louis Chambalon, 7 avril 1710).

15 Bail à loyer, 31 octobre 1708, ANQQ, Greffe de François Genaple. Gauvin et sa femme doivent en retirer un maximum de 300 livres pour les trois premières années et, pour les six dernières, soit entrer en société avec Rouer d'Artigny, soit retirer 500 livres par année. Gauvin se réserve aussi la «préférence» du travail à faire pour cette pêche.

16 Acte de société, 7 avril 1710, ANQQ, Greffe de Louis Chambalon. 
Le triumvirat est vite mis à l'épreuve. Au printemps de 1710, la première société entre Hazeur, Peire et les habitants étant légalement expirée, Jacques Gagnon et Étienne Bouchard, de leur propre chef, tendent une pêche à la pointe de la Rivière-Ouelle. Riverin, Philippe Peire et Rouer d'Artigny protestent auprès de l'intendant et obtiennent gain de cause sur ce point précis, mais doivent aussi sacrifier une bonne part de la substance de leur privilège. L'intendant interdit aux habitants de tendre à la pointe de la Rivière-Ouelle, devant la terre de Gauvin, car c'est là le site des entrepreneurs. Mais par contre, il leur permet de tendre devant leurs propres terres, pourvu que ces nouvelles pêches ne nuisent pas à celle déjà existante à la pointe de la Rivière-Ouelle. Voici le raisonnement de l'intendant dans son ordonnance du 16 avril 1710:

(...) attendu que ce droit exclusif allégué par le dit Sr Peyre n'est point un droit prohibitif aux autres habitants d'établir des pesches dans la devanture de leurs habitations ou les dits Sr Peyre et Riverin n'en ont point Etably; n'estant un droit exclusif suivant la permission qui leur a esté accordée, que pour les lieux ou ils voudroient l'établir en cas qu'il n'y en eut pas actuellement et non pas d'empêcher les habitants d'en Establir pendant le temps que durera leur permission, que cela ne peut estre entendu autrement puisques au lieu que par cette permission on a pretendu procurer un grand avantage à ce pays, Cela produiroit un effet tout contraire, parce que leurs entreprises ne réussissant point, ces fortes pesches ne s'établiroient jamais, au lieu que les habitants ayant lieu de les entreprendre sur eux dans les endroits qui ne seront Établis, il s'en trouvera quelqu'un qui réussira ce qui convient au Bien du pays, et par Conséquent aux Intentions de la Cour $(\ldots)^{17}$

Raudot est très clair. Le privilège des entrepreneurs ne s'applique qu'aux pêches existantes. Les habitants ont tout le loisir d'en tendre ailleurs. Ces limites imposées au privilège sont autant d'extension donnée aux droits des habitants. Mais par sa décision, l'intendant ne se pose pas en défenseur de ces derniers. S'il laisse cette latitude aux habitants, c'est uniquement dans le but d'assurer le développement et l'implantation massive de la pêche au marsouin dans le Bas Saint-Laurent. D'ailleurs, Raudot n'a pas de faveurs que pour les habitants. Devant les difficultés que Jacques Gagnon et consorts semblent continuer à causer à la pêche des entrepreneurs à la pointe de la Rivière-Ouelle, l'intendant force la continuation de la société de 1705 jusqu'à la fin du privilège en 1720 , enfermant de nouveau ces habitants dans un carcan dont ils se croyaient enfin débarrassés ${ }^{18}$.

Les conséquences de cette première manche juridique sont nombreuses. Elle éveille d'abord l'intérêt du seigneur de Rivière-Ouelle, le sieur de Boishébert. Celui-ci, appelé à reconnaitre le droit de pêche des

17 Ordonnance de Raudot, 16 avril 1710, ANQQ, OI, 4: f. 49

18 Ordonnance de Raudot, 20 mai 1710, ANQQ, OI, 4: f. 57. 
habitants en 1707, se rend compte sans doute qu'il ne peut jouir pleinement des ressources de sa seigneurie et que l'insouciance et la générosité de son père au sujet de la pêche l'ont placé dans une situation où il ne peut guère faire valoir ses droits. Aussi dès 1708, Boishébert cherche à obtenir la confirmation de la concession de la seigneurie de Rivière-Ouelle «avec faculté d'y establir une pesche de marsouins qu'il exploitera à ses frais et dépens» ${ }^{19}$. Occupé à sa carrière militaire, Boishébert délaisse ensuite la question de la pêche au marsouin jusqu'à la fin du privilège.

Également, la clarification des termes du privilège fait naître une nouvelle opposition qui vient cette fois des enfants du premier mariage de la femme de François Gauvin ${ }^{20}$. C'est au-devant de la terre de leur père défunt que se trouve la pêche des entrepreneurs et où travaillent Jacques Gagnon et consorts. Les habitants contestataires, Pierre Dancosse fils en tête, soutiennent que comme cette terre leur appartient en partie avec leur mère, ce sont eux qui devraient en faire valoir la devanture à la place de Jacques Gagnon et des autres. Ils estiment que leur droit de pêche a primauté sur la société formée par l'intendant. Ils ne contestent donc pas le privilège, mais la décision de Raudot qui met à leur place des habitants ne possédant pas la propriété des grèves exploitées pour la pêche. Après quelques incidents opposant le groupe de Dancosse à celui de Gagnon, l'intendant Bégon se voit forcé d'intervenir au printemps de $1714^{21}$. Il condamne les principaux belligérants (Dancosse, Gauvin et Gagnon) à 30 livres d'amende chacun et fait expressément défense de troubler la pêche des entrepreneurs à l'avenir. Surtout, il ne revient pas sur la décision de Raudot et laisse Gagnon et consorts s'occuper de la pêche de la pointe de la Rivière-Ouelle. Ces ordonnances semblent ramener le calme mais le groupe de Dancosse n'oubliera pas ses prétentions une fois le privilège expiré.

Enfin, l'ordonnance de 1710 permet à des habitants de tendre de nouvelles pêches. Des habitants de la Pointe-aux-Iroquois dans la seigneurie de Rivière-Ouelle ${ }^{22}$, qui avaient tendu dès 1709 , en profitent pour faire approuver leur sociétée ${ }^{23}$. L'apparition de tels concurrents, bien que légale, ne pouvait qu'inquiéter les entrepreneurs privilégiés.

\footnotetext{
19 M. le marquis Dalogny au Ministre, 1708, AC, C11A, 29: f. 227 (en marge).

20 Il s'agit de Pierre Dancosse fils, et des maris de ses soeurs, entre autres: Joseph Lizot, Pierre Bérubé et Jean Brisson. Ils semblent disposer de l'appui de François Gauvin, leur beaupère, et de Marie-Madeleine Bouchard, mère des Dancosse.

${ }_{21}$ Il est question de feux que Dancosse et sa parenté allument à la pointe de la RivièreOuelle et qui effraient les marsouins selon Gagnon, et d'une altercation pendant laquelle Gauvin, Dancosse et Gagnon «se sont attroupés les uns contre les autres». Voir: Ordonnances de Bégon, 22 mars et 23 avril 1714, ANQQ, OI, 6: f. 53v. et 63v.

${ }_{22}$ La Pointe-aux-Iroquois est située un peu au nord-est de la pointe de la Rivière-Ouelle et représente également un bon site pour la pêche au marsouin.

${ }^{23}$ Ces habitants sont: Jean Mignot, Louis Dubé, Jean Dechêne, Noël Pelletier, Jacques Bois et François Autin, tous voisins à la Pointe-aux-Iroquois; voir: Ordonnance de Raudot, 6 juin 1710, EO, III: 428 .
} 
Ceux-ci vont alors tenter de contrôler la pêche de la Pointe-aux-Iroquois. En 1713, Louis Rouer d'Artigny s'associe aux droits de trois des six parts de la pêche, tandis qu'en 1715, Philippe Peire parvient à faire de même pour les trois autres ${ }^{24}$. Malgré leur privilège légalement affaibli, les entrepreneurs n'abandonnent donc pas la partie. Jouissant d'une sorte de trève dans le cas de la pointe de la Rivière-Ouelle, ils contrent l'impact de l'implantation de nouvelles pêches ailleurs dans la région par une habile politique de récupération. La position des entrepreneurs n'est pas si mauvaise en 1720. Ils contrôlent les pêches à marsouins de la pointe de la Rivière-Ouelle, de la Pointe-aux-Iroquois, de l'islet SaintDenis à la Pocatière ${ }^{25}$, de la rivière des Caps et des îles de Kamouraska ${ }^{26}$. Ils ont donc réussi à colmater les brèches que l'ordonnance de 1710 avait causées. Leur position en est encore une de monopole dans le Bas Saint-Laurent.

\section{II- La fin du privilège, 1720-1730}

Les dernières années du privilège de 1705 semblent se passer dans l'attente de l'expiration de ce modus vivendi. Tous les intéressés se préparent en vue de faire valoir leurs droits en 1720. L'intendant Bégon va être sollicité par les réclamations de nombreux groupes et individus et il devra démêler un écheveau complexe de points légaux.

Dès l'automne 1717, Philippe Peire met ses droits de l'avant ${ }^{27}$. Il insiste sur les pertes de son frère dont le montant s'élevait à plus de 60000 livres en 1707 et sur ses propres dépenses pour l'amélioration de la pêche au marsouin. Mais en même temps que Peire, Boishébert réclame le privilège de cette pêche sur sa seigneurie. Il se base sur le fait que Peire, ayant refusé la succession de son frère, ne pouvait conserver le privilège de 1705 et devrait ainsi se le faire retirer en 1720 . Boishébert fait de plus valoir les dépenses de sa famille pour la mise en valeur de la seigneurie de Rivière-Ouelle (50 000 livres) dont les revenus sont bien faibles (300 livres par année) ${ }^{28}$. Boishébert mise également, quoique de façon très ambiguë, sur le droit seigneurial exclusif de pêche des poissons à lard; mais cet argument ne convainc nullement Vaudreuil et Bégon.

${ }^{24}$ Bail à ferme de Louis Dubé à Rouer d'Artigny, 15 octobre 1713; Société entre Rouer d'Artigny, J. Mignot et J. Bois, 2 novembre 1713; Société entre Philippe Peire, F. Autin, la veuve Pelletier et la veuve Dechêne, 18 mai 1715, ANQQ, Greffe de Louis Chambalon.

${ }_{25}$ Société entre Philippe Peire et Marie Gerbois de la Pocatière, 17 octobre 1715, ANQQ, Greffe de Louis Chambalon.

26 Vaudreuil et Bégon au Conseil de Marine, 14 octobre 1716, AC, C11A, 36: f. 29-29v.; les mêmes, 26 octobre 1719, AC, C11A, 40: f. 37-9.

27 A partir de 1715, on n'entend plus parler de Louis Rouer d'Artigny ni de Joseph Riverin comme associés au privilège.

28 Peire au Conseil de Marine, 26 octobre 1717, AC, C11A, 38: f. 27-28v.; Projet de mémoire au Roy (...), 23 mai 1719, AC, C11A, 40: f. 291v.; Vaudreuil et Bégon au Conseil de Marine, 26 octobre 1719, AC, C11A, 40: f. 37-9. 
En fait, après avoir pris connaissance des mémoires de Peire et de Boishébert, le gouverneur et l'intendant ont déjà arrêté leur décision, qu'ils vont envoyer à la Cour pour approbation. Ils décident que les deux candidats jouiront conjointement et par moitié du privilège de la pêche au marsouin et ce, pour une période de dix ans ${ }^{29}$. En 1720, Vaudreuil et Bégon définissent davantage le nouveau privilège:

\begin{abstract}
(...) les Srs Vaudreuil et Bégon estiment qu'il conviendroit qu'il fut expliqué dans le brevet pour ce nouveau privilège qu'ils /Peire et Boishébert/ jouiroient seulement des pesches dont jouit actuellement le S. Peire et qu'ils n'en pouroient Établir de nouvelles qu'au refus des seigneurs ou habitants des lieux audevant desquels ils voudroient les établir et qu'en rapportant par lesd. Srs. Peire et Boishébert les refus de ces seigneurs ou habitants, lesd. Srs de Vaudreuil et Bégon leur permettroient d'y faire ces nouveaux Établissements jusqu'à l'expiration de leur privilège y ayant de la justice que ceux dont les terres aboutissent sur le fleuve SaintLaurent ayent la préférence d'y Établir des pesches $(\ldots)^{30}$
\end{abstract}

Ainsi, l'ordonnance du 16 avril 1710, qui reconnaissait explicitement le droit de pêche des habitants, est confirmée et renforcée dix ans plus tard car le privilège de 1720 laisse encore moins de liberté à ses détenteurs. Ne sont privilégiées que les pêches déjà existantes et, pour en établir de nouvelles, Peire et Boishébert doivent obtenir le consentement des propriétaires du lieu en question. De cette façon, le droit de pêche et la propriété des grèves sont nettement mis au-dessus du privilège.

C'est encore à propos du site de la pointe de la Rivière-Ouelle qu'apparaissent des difficultés suite à la promulgation du nouveau privilège. Pierre Dancosse et ses beaux-frères reviennent à la charge afin d'obtenir la reconnaissance de leurs droits sur le site et la jouissance de son exploitation. Comme en 1714, ils s'en prennent à Jacques Gagnon et aux autres habitants qui y travaillent pour les entrepreneurs.

Bien déterminés à se voir donner raison et sûrs de leurs prétentions, Dancosse et consorts s'en remettent à l'intendant Bégon et n'ont aucun mal à le convaincre d'agir en leur faveur. Celui-ci constate en effet que les droits de Jacques Gagnon et consorts ne tiennent que par la société de 1705 qui est alors expirée. Or, les droits reconnus aux habitants ne sont que le droit de pêche sur une devanture leur appartenant. Dans ce cas, le groupe de Dancosse possède tous les atouts et Bégon n'hésite pas à lui rendre justice par deux ordonnances (17 mars 1720 et 15 mai 1721) qui le mettent en lieux et place de Gagnon et

\footnotetext{
${ }^{29}$ Ibid.

30 Vaudreuil et Bégon au Conseil de Marine, 26 octobre 1720, AC, C11A, 42: f. 28-28v.
} 
consorts pour l'exploitation de la pêche de la pointe de la RivièreOuelle $^{31}$.

Mais Dancosse et ses beaux-frères n'en restent pas là. Ils contestent globalement le «prétendu» privilège de Peire et de Boishébert sur la pointe de la Rivière-Ouelle. Ils affirment que c'est François Gauvin qui, le premier, a tendu une pêche à marsouins devant l'habitation de la veuve Dancosse, sa femme, à la pointe de la dite rivière, et, avec des voisins associés, a laissé entrer Pierre Peire et François Hazeur dans la société de 1705 . Ils ajoutent que ces «pauvres habitants, qui n'entendoient point les affaires», s'étaient fait rouler par les entrepreneurs lors de la rédaction des actes qui laissaient croire le contraire et que cette méprise devait être corrigée, maintenant qu'eux, les héritiers de Dancosse, étaient en mesure d'exploiter la pêche en question ${ }^{32}$. Dancosse et consorts s'élèvent donc contre l'existence même d'un quelconque privilège les forçant à partager la jouissance de la pointe de la RivièreOuelle. Implicitement, ils en demandent la révocation.

Bégon ignore ces dernières récriminations. Il croit que le fait de mettre Dancosse et ses beaux-frères à la place de Gagnon va arranger les choses. Il se trompait énormément. L'association entre Peire, Boishébert et le groupe de Dancosse, telle qu'ordonnée le 15 mai 1721, ne se réalisera jamais ${ }^{33}$. Pendant que les tenants du privilège cherchent à avoir gain de cause devant les tribunaux, le groupe de Dancosse ratifie une entente de 25 ans avec Frontigny et La Cetière pour l'exploitation de la pêche au marsouin à la pointe du nord-est de la Rivière-Ouelle ${ }^{34}$.

Ainsi, après 1721 , le privilège de la pêche au marsouin ne ressemble plus guère à un monopole. Légalement, on a vu à quel point le privilège est subordonné au droit de pêche des habitants ou des seigneurs dans le cas des nouvelles pêches à établir. Pratiquement, le privilège ne peut même plus prétendre assurer la jouissance des pêches déjà existantes. Dancosse et consorts, dans un geste qui en dit long sur leurs frustrations passées, sanctionnent la présence de nouveaux intermédiaires entre les habitants-pêcheurs et les marchés, privant ainsi les tenants du privilège du meilleur site de la région. Enfin, dans les années 1720 , le privilège va être confronté à une évolution de la pêche au marsouin qu'il ne pourra contrôler.

En effet, favorisée par une conjoncture de hauts prix ${ }^{35}$, la pêche au marsouin semble enfin en voie de se développer pleinement, selon le souhait des autorités coloniales et métropolitaines. Dans un premier

\footnotetext{
f. $63 \mathrm{v}$

Ordonnances de Bégon, 17 mars 1720 et 15 mai 1721, ANQQ, OI, 6: f. 357v. et 71/2:

Ordonnance de Bégon, 8 février 1721, ANQQ, OI, 71/2: f. 34.

Ordonnance de Bégon, 15 mai 1721, ANQQ, OI, 71/2; f. 63v.

Acte de société, 8 janvier 1722, ANQQ, Greffe de Étienne Janneau.

Voir note \# 3.
} 
temps, les pêches vont se multiplier un peu partout sur les deux rives de l'estuaire, là où le littoral le permet ${ }^{36}$. L'engouement pour la pêche au marsouin est tel qu'il se fait même des tentatives jusqu'à la côte de Beaupré et à l'île d'Orléans. La décentralisation de la pêche est donc complète: Peire et Boishébert ne sont plus que des exploitants parmi d'autres $^{37}$. Mais la pêche au marsouin elle-même prend un tournant décisif à cette époque. Loin de signifier le succès de la politique économique de l'État, le phénomène de la multiplication des pêches se traduit paradoxalement par une stagnation de la production. Les captures de marsouins se limitent bientôt à quelques unités par année, car le trop grand nombre de parcs, trop près les uns des autres, effraie l'animal qui n'ose plus s'approcher du rivage ${ }^{38}$. Le découragement s'empare alors de la plupart des exploitants. En 1730, personne ne va venir demander la prolongation du privilège ${ }^{39}$.

Dans les années 1730 et 1740 , la pêche au marsouin est devenue un mauvais souvenir pour les administrateurs et les entrepreneurs de Québec. Désormais réputée «casuelle» ${ }^{40}$, elle se fait toujours dans les meilleurs sites autour de Rivière-Ouelle, mais seulement comme activité d'appoint aux travaux agricoles.

\section{Conclusion}

Les grands gagnants des modifications successives apportées au privilège de la pêche au marsouin sont sans conteste les habitants de la région. Pendant ce processus, ils se garantissent une autonomie complète au plan local de l'exploitation des pêches. Cette autonomie découle naturellement de leur droit de pêche et contrebalance en quelque sorte leur très grande dépendance envers les intermédiaires en ce qui a trait à la fourniture d'outils et à la mise en marché de la production. En effet, le droit de pêche, s'il permet aux habitants de contrôler leur devanture, ne leur facilite évidemment pas l'accès aux réseaux commerciaux de l'époque et cela reste vrai en situation de monopole ou non. Le groupe de Pierre Dancosse, pourtant le plus radical, fournit un bel exemple en s'associant à cet effet à Frontigny et à La Cetière en 1722. Malgré cette limite, les habitants de Rivière-Ouelle ont la partie belle après 1720 . Ils ne sont pas ceux qui souffrent des déficiences de production de la pêche au marsouin. Contrôlant les meilleurs sites (pointe de la Rivière-

\footnotetext{
36 Les deux principales zones de concentration des parcs de pêche au marsouin se trouvent d'une part, dans le Bas Saint-Laurent, entre les Aulnaies et l'Isle-Verte, et d'autre part, sur la rive nord, à l'île-aux-Coudres et aux alentours. Voir les «états» de pêche au marsouin dans la série C11A (1721), 44: f. 94-97; (1724), 46: f. 269-269 v.; (1725), 47: f. 84-84v.; (1726), 48: f. 448$448 \mathrm{v} ;$; (1728), 50: f. 155-155v.

37 Ibid.

38 «Mémoire sur la pesche des marsouins», [Boishébert], 1726, AC, C11A, 48: f. 449-450.

39 Pour leur part, Boishébert commande au Détroit, tandis que Peire est affligé des «infirmités de la goutte». Beauharnois et Hocquart au Ministre, 12 octobre 1731, AC, C11A, 54: f. 144.

40 Beauharnois et Dupuy au Ministre, 20 octobre 1726, AC, C11A, 48: f. 52.
} 
Ouelle et Pointe-aux-Iroquois), les habitants ne cessent jamais de considérer la pêche au marsouin comme une activité d'appoint qui ne leur coûte rien, sinon du travail à temps perdu, et qui rapporte toujours quelque chose, bon an mal an.

Il convient aussi de retenir l'agressivité et la ténacité des habitants de la région qui font d'eux des partenaires économiques indociles pour l'État et les entrepreneurs. Par contre, cela crée également des frictions entre les habitants eux-mêmes. En effet, l'affirmation du droit de pêche des habitants de Rivière-Ouelle fait des perdants. Plusieurs associés de la première heure, tel Jacques Gagnon, qui n'ont aucun droit sur la pointe de la Rivière-Ouelle, bien qu'ils aient eux aussi le droit de pêche sur leurs propres terres, perdent en fait l'exploitation du meilleur site de la région en 1721. Entre Gagnon et Dancosse, il n'y a guère de sympathie, on le devine bien ${ }^{41}$.

Les entrepreneurs privilégiés, en plus de perdre le monopole d'exploitation dont ils rêvaient, vont peu à peu se désintéresser de la pêche au marsouin dans le courant des annnées 1720, quand il devient évident qu'en situation de libre concurrence, la production d'huile est fortement limitée. Une exploitation centralisée et monopolisée comme l'entendaient les détenteurs des privilèges aurait peut-être assuré de meilleurs résultats à long terme au niveau de l'ensemble de la production et des exportations d'huile de marsouin. Mais cela aurait pu se faire seulement si les entrepreneurs avaient eu la propriété des grèves du lieu choisi pour la pêche. En s'acharnant à vouloir faire de Rivière-Ouelle leur centre d'exploitation de la pêche au marsouin, les entrepreneurs sont allés au-devant des coups portés à leur privilège. Ils ont eu le tort de croire que leur monopole était intouchable grâce au soutien inconditionnel de l'administration coloniale.

Malheureusement pour les entrepreneurs, l'État ne voyait pas les choses du même oeill. Certes, l'administration coloniale épaule les entrepreneurs en leur accordant le privilège de la pêche au marsouin. Mais plutôt que de considérer cette permission comme un droit exclusif au seul bénéfice de ceux-ci et ne rapportant qu'indirectement à la vie économique coloniale, l'intendant se met en tête de se servir du privilège comme une incitation, un exemple à donner devant faire de la pêche au marsouin un secteur de production augmentant et diversifiant le commerce extérieur canadien. Dans ce sens, l'intendant ne peut admettre le cloisonnement de la pêche au marsouin dans le cadre étroit d'un monopole exclusif. Il faut au contraire que les pêches se multiplient, et les ordonnances de Raudot et de Bégon se fondent sur cet

$41 \quad$ Selon P.-H. Hudon, cette rivalité va persister longtemps à Rivière-Ouelle. Dans le cadre de mes recherches sur la colonisation de la région, je compte bien vérifier cette affirmation. Voir P.-H. Hudon, Rivière-Ouelle de la Bouteillerie. Trois siècles de vie, s. 1., Comité du Tricentenaire [1972], 495 p. 
objectif. Le privilège ne devient plus alors qu'une protection que l'État accorde aux premières pêches, une récompense aux premiers à avoir contribué à l'implantation de la pêche au marsouin dans le fleuve SaintLaurent ${ }^{42}$.

En dernier lieu, il faut souligner la mauvaise évaluation dont la pêche au marsouin a été l'objet dans les trente premières années du XVIII ${ }^{e}$ siècle. Il apparaît en effet que les espérances des entrepreneurs et de l'État aient été basées sur une surestimation du rendement possible de cette activité économique. En fait, quand on y regarde de près, on constate que la pêche au marsouin n'a jamais vraiment produit beaucoup, même avant $1720^{43}$. Les développements des années 1720 démontrent, de plus, qu'on connaît fort mal le marsouin et son comportement imprévisible. Pourtant, la pêche au marsouin s'est retrouvée faisant partie de l'arsenal économique dont les intendants ont voulu faire usage dans le premier quart du XVIII ${ }^{e}$ siècle. On se rend vite compte alors que si le Canada est une terre de «staples», ceux-ci n'y sont certes pas en quantité illimitée ni de qualité égale. La pêche au marsouin illustre très bien ces limitations et ces inégalités.

$42 \quad$ Desgroseilliers et Radisson avaient aussi obtenu un privilège de 20 ans pour la pêche du loup-marin et du marsouin dans les années 1670. Rien n'indique qu'ils en aient fait usage; J.-N. Fauteux, Essai sur l'industrie au Canada sous le Régime français (Québec, L.-A. Proulx, 1927), 2: $505-6$

${ }_{43}$ Voir l'annexe sur le rendement de la pêche au marsouin, compilée d'après toutes les informations trouvées dans la série $C 11 A$. Que valent ces chiffres? Â peu près rien si on les prend tels quels. Mais bien qu'inexacts, ils n'en donnent pas moins un certain ordre de grandeur qui établit les limites probables de la production. Enfin, si ces données avaient été délibérément diminuées (la plupart des renseignements sur la rive sud nous viennent des entrepreneurs), il serait pour le moins étrange que la pêche au marsouin ne suscite pas plus de convoitise après 1730 . 
ANNEXE

RENDEMENT DES PÊCHES AU MARSOUIN DANS LE ST-LAURENT, 1700-1740

\begin{tabular}{|c|c|c|c|}
\hline Années & Pêches & Marsouins & $\begin{array}{c}\text { Barriques } \\
\text { d'huile }\end{array}$ \\
\hline 1701 & de Vitré & plus de 60 & \\
\hline 1702 & & plus de $190(1)$ & \\
\hline 1706 & Hazeur \& Peire & «pas reussy» & \\
\hline 1707 & , & & 40 \\
\hline 1708 & , & & 100 \\
\hline 1716 & Peire 5-6 pêches & 23 & 14 \\
\hline 1717 & "’ & & 12 \\
\hline 1718 & , & & 22 \\
\hline & Petite-Rivière & 42 & 32 \\
\hline 1721 & $\begin{array}{l}\text { Rive Nord } 8 \\
\text { Rive Sud } 6\end{array}$ & $\begin{array}{c}160 \\
22\end{array}$ & 120 \\
\hline 1723 & Rive Sud & 4 & \\
\hline 1724 & Rive Nord 4 & 17 & \\
\hline & Rive Sud 13 & $\begin{array}{c}467 \text { pêches n'ont rien pris. } \\
\text { Les Sieurs Peire et Boishé- } \\
\text { bert ont «peu produit.» }\end{array}$ & \\
\hline 1725 & Rive Nord 4 & 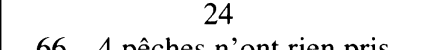 & \\
\hline 1726 & Rive Nord 9 & $\begin{array}{l}\text { 60 } 4 \text { peches } n \text { ont rien pris. } \\
46\end{array}$ & \\
\hline 1728 & $\begin{array}{l}\text { Peire et Boishébert } \\
\text { Rive Nord } 3 \\
\text { Rive Sud } 4\end{array}$ & $\begin{array}{c}\text { rien pris } \\
\text { plus de } 45 \\
30\end{array}$ & \\
\hline 1731 & $\begin{array}{c}\text { Rive Nord quelques } \\
\text { pêches }\end{array}$ & 14 & \\
\hline 1733 & $\begin{array}{c}\text { Rive Sud } 2 \\
\text { Île-aux-Coudres }\end{array}$ & $\begin{array}{c}0 \\
20\end{array}$ & \\
\hline & Rive Sud & 0 & \\
\hline 1734 & & «très petit objet» & \\
\hline 1735 & $\begin{array}{c}\text { Baie St-Paul } \\
\text { et Rivière--Ouelle }\end{array}$ & & \\
\hline 1736 & & $\begin{array}{l}\text { «Elle n'a pas esté plus avanta- } \\
\text { geuse cette année que les précé- } \\
\text { dentes» }\end{array}$ & \\
\hline 1737 & $\begin{array}{c}\text { Baie St-Paul } \\
\text { Kamouraska et Isle- } \\
\text { Verte }\end{array}$ & & 50 \\
\hline 1741 & Tadoussac & & $80(2)$ \\
\hline
\end{tabular}

1 Callières et Beauharnois écrivent que le Sieur de Vitré et ses associés ont «déjà pris plus de 250 marsouins»; on peut en soustraire les 60 pris en 1701; AC, C11A, 20: f. 63.

2 Cet établissement à Tadoussac est géré par Cugnet, directeur du Domaine. Après les mauvaises expériences de pêches au marsouin de ce dernier entre 1720-3, il peut sembler étrange de le retrouver impliqué à nouveau dans une telle entreprise. Mais certaines références de Hocquart sur les activités de Cugnet à Tadoussac, surtout en ce qui concerne la fabrication de la colle de poissons, laisse entendre que la pêche au loup-marin y était sans doute dominante. Les 80 barriques en question ici sont sans doute de l'huile de loup-marin. cf. Hocquart au Ministre, 28 oct. 1741, AC, C11A, 76: f. 29-29 verso; aussi A.J.E. Lunn, Economic Development in New France, (unpublished PH.D.thesis) (McGill University, 1942), 212.

Source: AC, C11A. 